\title{
Criterios de calidad en diagnóstico colposcópico
}

\author{
Antonio González Mazuelo*; Luis Guillermo Echavarría R.**
}

RESUMEN OBJETIVO: Tuvimos tres indicadores como parámetros de calidad en el diagnóstico colposcópico: Correlación colpohistológica, porcentaje de conizaciones diagnósticas y seguridad diagnóstico-terapéutica.

Quisimos obtener dichos indicadores en nuestra propia experiencia en el Centro de Patología cérvico-uterina del Instituto Metropolitano de Salud de Medellín.

MATERIAL Y METODOS: En el período de junio de 1991 a junio de 1993 se evaluaron 484 pacientes con citología anormal. la correlación colpo-histológica fue del $\mathbf{8 2 . 1 \%}$, el porcentaje de conizaciones diagnósticas del $2.9 \%$ y la seguridad diagnóstico-terapéutica del 99.15\%.

CONCLUSION: Estos resultados muestran una calidad aceptable en el diagnóstico colposcópico. Proponemos a los ginecólogos que trabajan en colposcopia dichos indicadores de control de calidad.

PALABRAS CLAVES: Parámetros de calidad, colposcopia, citología, histología.

SUMMARY OBJECTIVE: We have three points as parameter of quality of the colposcopic examination: Correlation among colposcopy and histology, percentage of diagnostic conization and safety among diagnosis and therapy.

Obtain this parameter in our experience in the center of cervical pathology of the Institute Metropolitan of Health of Medellin.

MATERIAL AND METHODS: In the period of June 1991 to June 1993 we examinated 484 patients with abnormal cytology. Result: the correlation among colposcopy and histology was $82,1 \%$, the percentage of diagnostic conization was $2,9 \%$ and the safety among diagnosis and therapy was $99,15 \%$.

CONCLUSION: This result shows a quality acceptable of the colposcopic examination. Propose to the center or individual whom work in colposcopy, this parameter of control of quality.

KEY WORDS: Parameter of quality, colposcopy, cytology, histology.

\section{Introducción}

A raíz del establecimiento en Colombia del programa de detección y control del carcinoma de cuello uterino por parte de Minsalud y el Instituto Nacional de Cancerología, en mayo de 1991 se creó una Unidad de Patología Cérvico-uterina en el Instituto Metropolitano de Salud de Medellín. Desde el inicio de actividades al grupo de Ginecólogos del Centro ha mantenido un interés permanente por garantizar una buena calidad de atención. De allí surgió la necesidad de tener algunos indicadores que nos permitieran medir, con bases objetivas, la calidad del diagnóstico colposcópico.

La colposcopia es una técnica diagnóstica de amplia aceptación mundial para el estudio de las pacientes con citología cérvico-uterina anormal. A pesar de la capacitación y experiencia del examinador, o de pronto en virtud de la misma, el grado de subjetividad del examen en sí no permite siempre una aproximación exacta al

\footnotetext{
* Médico Ginecobstetra. Sistema Comunal de Servicios de Salud de Castilla.

** Médico Ginecobstetra. Instituto Metropolitano de Salud de Medellín.
}

mayor grado de lesión epitelial presente en el cérvix de la paciente, la subjetividad del examen, de la cual no podemos despojarnos, evita que nuestra impresión a través del colposcopio coincida siempre con el diagnóstico del patólogo y, lo que es más importante, con la lesión más avanzada presente en la paciente. Diversos autores reconocen una aproximación máxima del $85 \%$.

En virtud de lo anterior la objetividad de la colposcopia podrá medirse según unos parámetros de control de calidad.

\section{Materiales y métodos}

Las pacientes evaluadas en el Centro tienen historia clínica completa y son remitidas desde los Centros y Puestos de Salud con resultados anormales en la citología.

El examen colposcópico queda consignado en un formato de variables cerradas donde se incluye la terminología adoptada en el último Congreso de la Federación Internacional de Patología Cervical y colposcopia.

El Centro cuenta con un colposcopio Ciss-Gena de 5 aumentos y filtro verde. 
Todo el proceso diagnóstico terapéutico está protocolizado según normas del Instituto Nacional de Cancerología y la información se consolida semestralmente en formularios especiales de donde se tomaron los datos.

Tomamos de la literatura tres indicadores que proponemos como criterios de calidad diagnóstica en colposcopia: Uno, Correlación colpo-histológica. Dos, porcentaje de conizaciones diagnósticas y tres, seguridad diagnóstico-terapéutica.

El primero tiene que ver con el grado de coincidencia entre el diagnóstico colposcópico y el resultado de la biopsia dirigida. Se adoptó el esquema propuesto por Dexeus (1) muy cercano al de Coppleson (2). En cuatro categorías reconociendo que la colposcopia tiene un lenguaje independiente del histológico. En colposcopia hablamos de zona de transformación normal o anormal; la anormal puede ser: Poco significativa (PS), significativa (S), altamente significativa (AS), o invasión. La tabla No. 1 muestra la equivalencia histológica; decimos que hay correlación cuando la impresión colposcópica y la histología coinciden según las equivalencias planteadas.

Tabla 1

EQUIVALENCIA ENTRE LA IMPRESION COLPOSCOPICA Y LA BIOPSIA DIRIGIDA (*)

\begin{tabular}{|l|l|}
\hline Colposcopia & Histología \\
\hline Zona de transformación & Biopsia \\
\hline Poco significativa & $\begin{array}{l}\text { Metaplasia } \\
\text { Condiloma viral } \\
\text { NIC. I }\end{array}$ \\
\hline Significativa & NIC. II \\
\hline Altamente significativa & NIC. III \\
\hline Invasión & Infiltrante \\
\hline
\end{tabular}

(*) (Tomado de Dexeus. S.) (1).
El segundo indicador es el porcentaje de conizaciones diagnósticas realizadas al grupo de pacientes por no correlación cito-histológico o colposcopias no satisfactorias; excluimos aquellos conos que se realizan para clasificar tumores microinvasores.

La seguridad diagnóstico-terapéutica se refiere a que siempre el espécimen quirúrgico-terapéutico (cono o histerectomía) debe mostrar una lesión igual o menor a la establecida en la biopsia dirigida por colposcopia. La calidad del diagnóstico colposcópico es aceptable cuando la correlación colpo-histológica es superior al $80 \%$ (3), el porcentaje de conizaciones diagnósticas oscila entre el 0 y el 6\% (4) y la seguridad diagnóstico-terapéutica equivale al $100 \%$.

\section{Resultados}

Durante el período de junio de 1991 a junio de 1993 fueron evaluadas en el Centro de Patología Cérvicouterina del Instituto Metropolitano de Salud de Medellín un total de 484 pacientes con citología anormal.

Cuatrocientas sesenta y siete pacientes $(96.5 \%)$ provenían de Medellín y 17 pacientes (3.5\%) de otros Municipios cercanos.

El $58.9 \%$ de las pacientes tenían edades comprendidas entre los 25 y los 45 años, $20.9 \%$ eran menores de 25 años y $20.2 \%$ eran mayores de 45 años.

La evaluación completa de estas pacientes requirió de 524 exámenes colposcópicos; en el $8.2 \%$ fue necesario un segundo examen.

De las 484 colposcopias iniciales, 423 fueron satisfactorias y $61(12.6 \%)$ no satisfactorias o sin lesión visible; en estas últimas se hizo curetaje endocervical dando positivo el $19.7 \%$ de los casos.

En 16 pacientes no fue posible conocer el resultado de la biopsia. Se hizo diagnóstico histológico en 468 pacientes. Doscientas trece pacientes $(45.5 \%)$ presentaron cervicitis crónica y/o infección por papiloma virus humano; 70 pacientes (15\%) tenían NIC I, 57 pacientes (12.2\%) NIC II, 104 pacientes (22.2\%) NIC III y 24 pacientes $(5.1 \%)$ tumores infiltrantes.

Tabla 2

CORRELACION COLPOHISTOLOGICA EN 468 CASOS EN EL CENTRO DE PATOLOGIA CERVICOUTERINA DEL INSTITUTO METROPOLITANO DE SALUD ENTRE 1991 Y 1993

\begin{tabular}{|l|c|c|c|c|c|}
\hline $\begin{array}{l}\text { Biopsia } \\
\text { Colposcopia }\end{array}$ & Negativo & $\begin{array}{c}\text { Metaplasia } \\
\text { PVH } \\
\text { NIC. I }\end{array}$ & NIC. II & NIC. III & Invasión \\
\hline Normal & 33 & 17 & 2 & 0 & 0 \\
\hline PS - & 10 & 186 & 9 & 7 & 0 \\
\hline S & 8 & 23 & 41 & 8 & 2 \\
\hline AS & 0 & 6 & 4 & 84 & 13 \\
\hline Invasión & 0 & 0 & 1 & 5 & 24 \\
\hline Total & 51 & 232 & 57 & 104 & 9 \\
\hline
\end{tabular}

Nota: Las áreas sombreadas equivalen a los casos con correlación colpohistológica. 
Las pacientes fueron tratadas por diversos métodos así:

211 con tratamientos médicos, 110 con crioterapia, 117 con cirugía (49 conizaciones y 68 histerectomías) y 30 pacientes fueron remitidas a otros centros de nivel superior.

La correlación colpo-histológica fue adecuada en 384 pacientes $(82.1 \%)$ la impresión colposcópica resultó mayor que la biopsia en 37 casos (7.9\%) y menor en 47 casos $(10 \%)$ (tabla No. 2).

En total se realizaron 25 conizaciones diagnósticas (5.2\%) pero sólo 14 de ellas $(2.9 \%)$ por falta de correlación citohistológica o colposcopia no satisfactoria con curetaje endocervical positivo, las restantes conizaciones se hicieron para excluir o clasificar tumores microinvasores.

Sobre 117 cirugías con intención terapéutica sólo una paciente mostró en la pieza quirúrgica una lesión mayor que la diagnosticada inicialmente con base en la colposcopia. La paciente tenía diagnóstico de NIC III con colposcopia no satisfactoria y curetaje endocervical negativo. El análisis del espécimen (histerectomía simple ampliada) mostró un tumor microinvasor YA2 FIGO con invasión entre 3 y $5 \mathrm{~mm}$. de profundidad y tuvo que ser remitida para radioterapia complementaria. La seguridad diagnóstico-terapéutica se cumplió en 116 casos $(99.15 \%)$.

\section{Discusión}

El establecimiento en Colombia del programa de Detección y Control del carcinoma de cuello uterino ha creado un significativo número de Centros de diagnóstico colposcópico en todo el país dirigido por personal médico no subespecializado en Oncología Ginecológica. Lo anterior exige que la capacitación recibida deba ser complementada con la experiencia diaria pero ésta última no perfeccionará nuestros conocimientos sin un control riguroso de la actividad realizada.

Se hace necesario entonces tener unos patrones o stándares de cuya evaluación permanente podamos deducir la calidad y seguridad de nuestro trabajo diagnóstico.

Una condición inicial es comentar un lenguaje común. La evaluación colposcópica debe quedar consignada siguiendo los lineamientos de la revisión más reciente de la Federación Internacional de Patología Cervical y Colposcopia (5): en base a ella rediseñamos el formato y todos los Ginecólogos del Centro consignan en él sus hallazgos siguiendo una misma terminología. La conjunción de hallazgos colposcópicos debe configurarse en un diagnóstico, el cual debe hacerse el lenguaje colposcópico y no histológico.

Coppleson (2) en 1960 fue el primero que propuso graduar las zonas de transformación anormales en cuatro categorías según los aspectos cualitativos (contorno, densidad, localización, etc.) de las lesiones observadas.

Reid (6) diseñó un sistema semicuantitativo con asignación de puntaje para definir categorías muy semejantes.

Recientemente (7) ambos autores han perfeccionado sus propuestas con algunas modificaciones pero conservando los mismos principios.

Dexeus (1) en literatura española sigue una línea semejante.

Las categorías colposcópicas varían en sus equivalencias histológicas pero todaș han probado un alto grado de precisión diagnóstica.

El grupo canadiense de la Columbia Británica en el mismo afán de establecer unos criterios de calidad colposcópica propuso una cifra del $80 \%$ de equivalencia entre el diagnóstico histológico y la impresión colposcópica (3).

En esta experiencia una cifra del $82.1 \%$ es aceptable.

Barros y colaboradores (8) hablaron de seguridad diagnóstica en colposcopias; en su artículo sobre 367 pacientes sometidas a cirugía, sólo en una paciente se pasó por alto un carcinoma invasor. Resaltan la importancia de no omitir nunca el curetaje endocervical en casos de colposcopia no satisfactoria y si éste es positivo, realizar siempre conización diagnóstica. Cuando el curetaje es negativo, es excepcional que la paciente porte un carcinoma invasor.

Urcuyo (9) informa de un caso en 117 pacientes con colposcopia no satisfactoria y curetaje endocervical negativo $(0.85 \%)$.

La seguridad diagnóstica del grupo de Barros equivale al $99.73 \%$.

En nuestra experiencia $99.15 \%$ es aceptable.

Otro objetivo importante de la colposcopia será siempre reducir al mínimo la necesidad de realizar conizaciones diagnósticas. Este es otro parámetro importante y constituye uno de los progresos más importantes de la colposcopia en el manejo de la patología cervical. Dos autores informan cifras de 3 y $3.1 \%$ de conizaciones diagnósticas (10-11). Barros obtuvo $2.7 \%$.

$2.9 \%$ de conizaciones en nuestro Centro es una cifra que coincide con la literatura.

\section{Conclusiones}

Definimos tres parámetros para evaluar la calidad y seguridad del diagnóstico colposcópico.

Los resultados obtenidos en la evaluación de 484 pacientes mostraron una calidad aceptable en la experiencia del Centro de Patología cervical del Instituto Metropolitano de Salud de Medellín.

Proponemos a los grupos que trabajan en colposcopias el uso de estos indicadores para el control de calidad. 


\section{BIBLIOGRAFIA}

1. Dexeus S. Tratado y Atlas de Patología Cervical. Editorial Salvat. Barcelona, 1989; 93.

2. Coppleson $\mathrm{N}$ et al. Colposcopy: A scientific and practical approach to the cervix, vagina and vulva in health and disease. Ed. Springfield I.L. 1986.

3. Benedet JL et al. A quality-control program for colposcopic practice. Obstet. and Gynecol. 1991; 78(5): 872.

4. Boelter WC. Correlation between colposcopic gradimg directec punch biopsy and conizacion. Am. J. Obstet. Gynecol. 1975; 122(8): 945.

5. Stalf A., Wilbamks GD. An international terminology of colposcopy report of the nomenclature committee of international federation of cervical pathology and colposcopy. Obstet and Gynecol. 1991; 77: 313.

6. Reid R., Scalzi P. Genital warts and cervical cancer. An improved colposcopic index for differentiating benign papilloma viral infections for high grade cervical intraepitelial neoplasia. Am. J. Obstet. Gynecol. 1985; 153: 611 .

7. Wrioht VC. Obstetrics and ginecology clinics of North America. Contemporary colposcopy. 1993 20(1): 100, 146.

8. Barros F. y otros. Evaluación de la colposcopia en el estudio de las pacientes con neoplasia cervical. Revista Colombiana de Obstetricia y Ginecología. 1992; 43(3): 208.

9. Urcuyo R et al. Some observations on the valve of endocervical curettage performed asonn integral part with abnormal cervical citology. Am. J. Obstet. Gynecol. 1977; 128: 787.

10. Hatch KD et al. Role of endocervical curettage in colposcopy. Obstet. Gynecol. 1985; 65: 403.

11. Javaheri G., Fedgin MD. Diagnostic valve of colposcopy in the investigation of cervical neoplasia. Am. J. Obstet. Gynecol. 1980; 137: 588 